\title{
The use of neutrophil-to-lymphocyte ratio (NLR) as a marker for COVID-19 infection in Saudi Arabia
}

\author{
A case-control retrospective multicenter study
}

Anwar A. Sayed, MBBS, PhD, Assem A. Allam, MBBS, MD, Ayman I. Sayed, MBBS, Mohammed A. Alraey, MBBS, MD, Mercy V. Joseph, BSN, CIC.

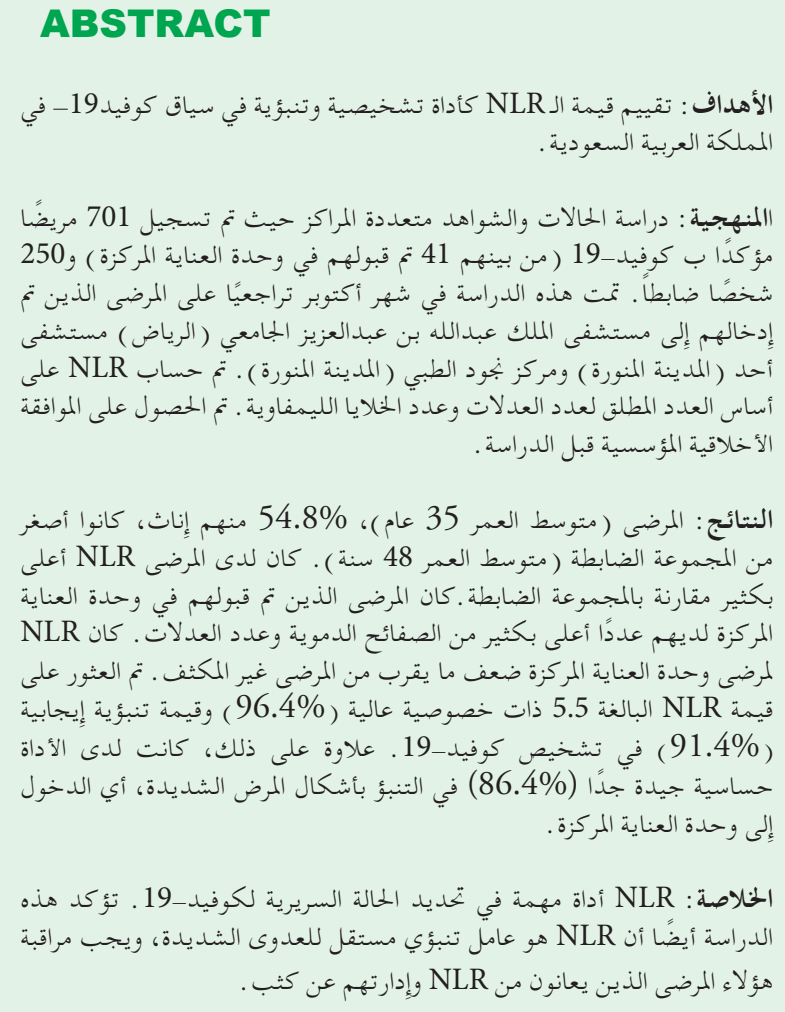

Objectives: To assess the neutrophil-to-lymphocyte ratio (NLR) diagnostic and prognostic value in the context of Coronavirus disease-2019 (COVID-19) infection in Saudi Arabia.

Methods: A case-control study in which 701 confirmed COVID-19 patients (of which 41 were intensive care unit [ICU]-admitted) and 250 control subjects were enrolled. The study was conducted retrospectively in October on patients admitted to 3 separate hospitals in Saudi Arabia namely: King Abdullah Bin Abdulaziz University Hospital (Riyadh), Ohud Hospital (Madinah), and Nojood Medical Center (Madinah) between May and September 2020. Neutrophil-to-lymphocyte ratio was calculated based on absolute neutrophil and lymphocyte count. Institutional ethical approval was obtained prior to the study.

Results: Patients (median age 35 years), of which 54.8\% were females, were younger than the control cohort (median age 48 years). Patients had significantly higher NLR compared to the control group. Intensive care unit admitted patients had significantly higher platelet, WBC and neutrophil counts. The ICU patients' NLR was almost twice as of the non-intensive patients. The NLR value of 5.5 was found to be of high specificity (96.4\%) and positive predictive value $(91.4 \%)$ in diagnosing COVID-19. Furthermore, it had a very good sensitivity $(86.4 \%)$ in predicting severe forms of disease, such as, ICU admission.

Conclusion: Neutrophil-to-lymphocyte ratio is an important tool in determining the COVID-19 clinical status. This study further confirms the prognostic value of NLR in detecting severe infection, and those patients with high NLR should be closely monitored and managed.

Keywords: complete blood count, COVID-19, NLR, prognosis, Saudi Arabia

Saudi Med J 2021; Vol. 42 (4): 370-376

doi: 10.15537/smj.2021.42.4.20200818

From the Department of Medical Microbiology and Immunology (Sayed AA), Faculty of Medicine, Taibah University; from the Department of Internal Medicine (Sayed AI), Nojood Medical Center, Madinah; Department of Infection Control (Allam, Alraey, Joseph), King Abdullah Bin Abdulaziz University Hospital, Riyadh, Kingdom of Saudi Arabia; and form the Department of Surgery and Cancer (Sayed AA), Imperial College London, London, United Kingdom.

Received 31st December 2020. Accepted 22nd February 2021.

Address correspondence and reprint request to: Dr. Anwar A. Sayed, Department of Medical Microbiology and Immunology, Faculty of Medicine, Taibah University, Madinah, Kingdom of Saudi Arabia. E-mail:dsayed@taibahu.edu.sa

ORCID ID: https://orcid.org/0000-0002-2243-0971 
$\mathrm{T}$ he novel coronavirus disease-2019 (COVID-19) global pandemic has resulted in efforts of researchers and governments being devoted to understanding this novel infection and how to prevent its spread. Gaining insights on the infection and understanding its effect on the body and the immune system will allow for the development of precise diagnostic assays for COVID-19.

Although it was first described as a respiratory disease; however, up to $20 \%$ of COVID-19 patients experience severe infection with severe extra-pulmonary manifestations including coagulopathy and septic shock. ${ }^{1}$ These manifestations are often unexpected, rapid, and fatal if not managed urgently in an intensive care unit (ICU). ${ }^{2}$ Additionally, COVID-19 patients had significantly high levels of pro-inflammatory cytokines, secondary to excessive immune response, and is often referred to as a "cytokine storm". 3,4 Such findings further support the systematic nature of the infection and hence developing diagnostic and prognostic tools should not focus solely on the respiratory system. These developed diagnostic tools varied in their detecting mechanism. Several diagnostic assays have been developed which are based on detecting anti-COVID-19 immunoglobulin, as well as assays that detect viral particles, such as, quantitative and real-time polymerase chain reaction (PCR). So far, the PCR methods remain the most reliable and accepted method of diagnosis for COVID-19 infection. ${ }^{5}$

Several independent risk factors have been determined to yield poorer outcomes when present among patients with COVID-19, including older ages, obesity and the presence of comorbidities, such as diabetes mellitus. ${ }^{6}$ It has been suggested that neutrophilto-lymphocyte ratio (NLR) carries a prognostic value in a variety of conditions including, but not limited to, acute respiratory distress syndrome, solid tumors and sepsis..$^{7-9}$ Additionally, studies have suggested that NLR is an independent prognostic indicator for the severity of COVID-19 infection. ${ }^{10}$ However, the importance of NLR, in regard to the COVID-19 infection, as a diagnostic, such as, to differentiate from those who are not infected, and prognostic tool remains to be determined in Saudi Arabia.

Disclosure. Authors have no conflict of interests, and this research was funded by the Deanship of Scientific Research, Princess Nourah bint Abdulrahman University, Riyadh, Kingdom of Saudi Arabia through the Fast-track Research Funding Program.
This study aims to assess the value of NLR as a diagnostic tool, in a cohort of patients with COVID-19 in Saudi Arabia, and its usefulness in predicting poorer outcomes.

Methods. Nine hundred fifty-one participants were included in this study from 3 separate hospitals in Saudi Arabia namely: King Abdullah Bin Abdulaziz University Hospital (Riyadh), Ohud Hospital (Madinah) and Nojood Medical Center (Madinah). There were 701 patients admitted for a suspected COVID-19 infection, and diagnosis was confirmed by quantitative PCR (qPCR) on collected nasopharyngeal swab. The inclusion criteria for this study are in line with the Saudi Ministry of Health criteria for hospital admission of COVID-19 patients v1.1, ${ }^{11}$ which are as follows: confirmed cases by qPCR, symptomatic, low oxygen saturation $<94 \%$ on room air and clinical or radiological evidence of pneumonia. Patients with other criteria indicating admission such as the use of biological immunosuppressants, active malignancy or history of organ transplant were excluded from this study. Out of these 701 patients, 41 patients were admitted to the ICU. Intensive care unit admission criteria were as follows: a temperature of $38.5^{\circ} \mathrm{C}$ accompanied with a decrease in mean arterial pressure below $60 \mathrm{mmHg}$ or shortness of breath that is New York Heart Association (NYHA) class III or higher which is characterized as "marked limitation in activity due to symptoms, even during less-than-ordinary activity, example: walking short distances (20-100 m). Comfortable only at rest." ${ }^{\text {I }}$

The remaining 250 subjects served as COVID-19free control subjects who were admitted for an elective surgical procedure and tested negative for COVID-19 using qPCR diagnostic test.

A multicenter case-control cross-sectional study in which patients' clinical data were anonymously collected. This study was conducted in October retrospectively on patients admitted between May and September 2020. Collected data included patients' gender, age and complete blood count results with white blood cell (WBC) differentials. No personal or identification information were collected in this study. This study was conducted after obtaining the ethical approval no. 005-1442 from the Taibah University College of Medicine Research Ethics Committee, Madinah, Saudi Arabia

Statistical analysis. Shaprio-Wilk test was used to analyze the data distribution of the participants, demonstrating a nonparametric distribution. The NLR calculation was based on their absolute count and compared between controls and COVID-19 patients. 
Nonparametric methods were used throughout the study such as Mann-Whitney u-test to compare variables between the cohorts. The values of NLR use, such as the sensitivity as a COVID-19 indicator were calculated according to the median patients' NLR values. Statistical significance were set at a $p$-value of $<0.05$. The statistical analysis was carried out using GraphPad Prism 9 (GraphPad Software, California, United States).

Results. Nine hundred fifty-one participants were included in this study; 701 of which are confirmed COVID-19 cases. There were 384 (54.8\%) female patients and $317(45.2 \%)$ male patients, whereas the control cohort included 112 female participants (44.8\%) and 138 male participants (55.2\%). The median patients' age was 35 years old (95\% CI: 34.87-37.58) which was significantly younger than the control cohort (48 years old, 95\% CI: 44.58-48.13). The summary of the participants' characteristics are presented in Table 1.

Our cohort of patients had a median red blood cells (RBC) count of $4.75 \times 10^{6} / \mathrm{L}$ which was significantly lower compared to the control group $(p<0.0001)$. Although the median hematological values of the COVID-19 patients did not suggest an anemic profile, patients had significantly lower hemoglobin levels, mean corpuscular volume (MCV) and mean corpuscular hemoglobin $(\mathrm{MCH})$ compared to the control cohort $(p<0.0001)$. Furthermore, COVID-19 patients had significantly lower platelet count compared to the control group (231.5 versus [vs] $309.5 \times 10^{3} / \mu$; $p<0.0001)$.

Upon examining the differential (WBC) profile, many differences were observed between the COVID-19 cohort and the control group. Patients with COVID-19, in comparison to the control group, had significantly lower WBC indices, including total WBC count (5.5 vs $\left.7.2 \times 10^{3} / \mu \mathrm{l}\right)$, neutrophil count (3.48 vs $\left.4.4 \times 10^{3} / \mu \mathrm{l}\right)$, lymphocyte count $(0.9$ vs 2.1 $\left.\mathrm{x} 10^{3} / \mu \mathrm{l}\right)$ and monocyte count $(0.33$ vs 0.6$)$. All these differences were statistically significant $(p<0.0001)$. However, COVID-19 patients had significantly higher neutrophil percentages compared to the control cohort (66.8 vs $58 \% ; p<0.0001$ ). Despite the lower WBC indices in COVID-19 patients, the calculated NLR was significantly higher compared to the control group (2.9 vs $2.18 ; p<0.0001$ ) as shown in Figure 1.

To examine whether the NLR correlated with any of the patients' biological variables, multiple linear regression analysis was performed. Expectedly,

Table 1 - Clinical characteristics and laboratory results of the control and Covid-19 cohorts.

\begin{tabular}{|c|c|c|c|}
\hline Characteristics & $\begin{array}{l}\text { Controls }(\mathrm{n}=250) \\
\text { median }(95 \% \text { CI })\end{array}$ & $\begin{array}{l}\text { COVID-19 cases }(\mathrm{n}=701) \\
\text { median }(95 \% \mathrm{CI})\end{array}$ & $P$-value \\
\hline \multicolumn{4}{|l|}{ Gender, $n$ (\%) } \\
\hline Male & $138(55.2)$ & $317(45.2)$ & \multirow{2}{*}{$0.0079^{*}$} \\
\hline Female & $112(44.8)$ & $384(54.8)$ & \\
\hline Age (years) & $48(44.58-48.13)$ & $35(35.09-37.77)$ & $<0.0001^{*}$ \\
\hline Red blood cell x $10^{6} / \mathrm{L}$ & $5.08(5.02-5.11)$ & $4.75(4.65-4.78)$ & $<0.0001^{*}$ \\
\hline Hemoglobin g/dL & $14(13.6-14.06)$ & $13.2(11.79-12.52)$ & $<0.0001^{*}$ \\
\hline Mean corpuscular volume fL & $90.35(88.74-90.8)$ & $86.35(84.98-86.33)$ & $<0.0001^{*}$ \\
\hline Mean corpuscular hemoglobin pg & $29.55(29.28-29.69)$ & $28.7(27.96-28.49)$ & $<0.0001^{*}$ \\
\hline $\begin{array}{l}\text { Mean corpuscular hemoglobin concentration } \\
\mathrm{g} / \mathrm{dL}\end{array}$ & $33(32.91-33.14)$ & $33(32.51-33.02)$ & 0.63 \\
\hline Platelet count $\times 10^{3} / \mu \mathrm{l}$ & $309.5(297.5-320.7)$ & $231.5(242.2-260.8)$ & $<0.0001^{*}$ \\
\hline White blood cell count $\times 10^{3} / \mu \mathrm{l}$ & $7.2(6.92-7.37)$ & $5.5(6.12-6.83)$ & $<0.0001^{*}$ \\
\hline Neutrophil count $\times 10^{3} / \mu \mathrm{l}$ & $4.4(4.28-4.64)$ & $3.48(4.09-4.75)$ & $<0.0001^{*}$ \\
\hline Neutrophil percentage & $58(56.03-58.6)$ & $66.8(63.35-66.47)$ & $<0.0001^{*}$ \\
\hline Lymphocyte count $\times 10^{3} / \mu \mathrm{l}$ & $2.1(1.95-2.1)$ & $0.9(0.9-1.31)$ & $<0.0001^{*}$ \\
\hline Lymphocyte percentage & $30.45(29.8-31.31)$ & $22.6(22.88-25.61)$ & $<0.0001^{*}$ \\
\hline Monocyte count $\times 10^{3} / \mu \mathrm{l}$ & $0.6(0.61-0.69)$ & $0.33(0.33-0.61)$ & $<0.0001^{*}$ \\
\hline Neutrophil-to-lymphocyte ratio & $2.18(2.3-2.6)$ & $2.9(4.24-5.31)$ & $<0.0001^{*}$ \\
\hline
\end{tabular}

The table demonstrates the clinical characteristics and the complete blood results of both the control and patients' cohorts. Laboratory results are stated as median values with $95 \%$ confidence intervals between brackets, unless stated otherwise. ${ }^{*}$ Statistical significance. CI: confidence intervals 


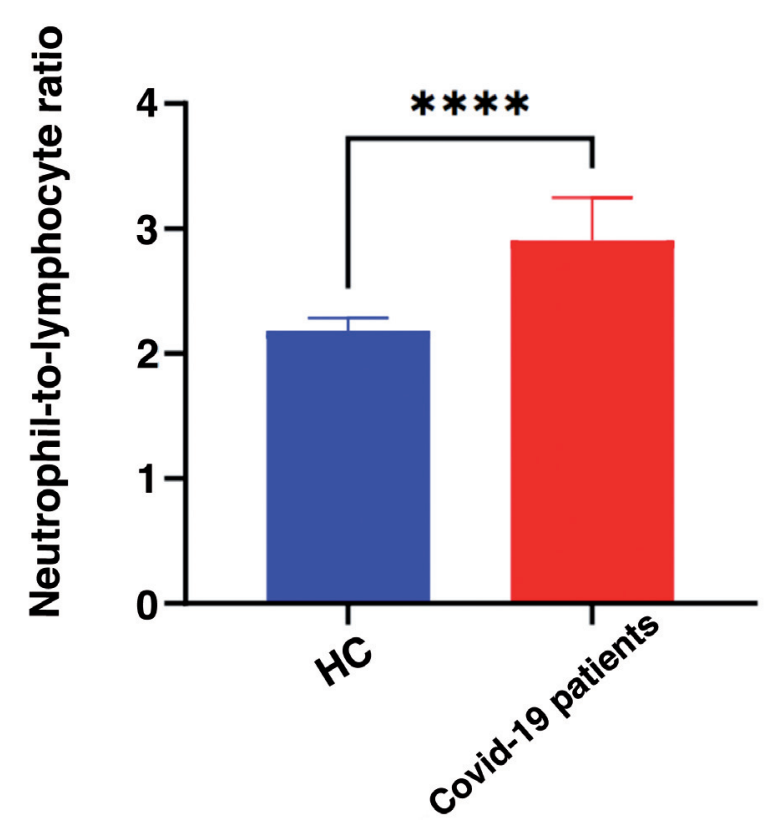

Figure 1 - Comparisons of neutrophil-to-lymphocyte ratios (NLR) between the control and COVID-19 cohorts. The bar charts demonstrate the differences of the median NLR between the control group (blue) and of the COVID-19 patients (red) which is significantly higher in the latter. HC: healthy control, $* * * *$ denotes a $p<0.0001$ neutrophil and lymphocytic count significantly correlated with NLR $(p<0.0001)$. However, none of the patients' age, RBC indices nor the platelet count correlated significantly with the NLR, suggesting that NLR is an independent indicator of disease activity.

From the total patients' cohort, 41 of them were admitted to the ICU. The summary of the ICU patients' characteristics is presented in Table 2.

Out of these ICU patients, 22 patients were male $(53.7 \%)$ while the remaining $46.3 \%$ were female. Their median age was 45 years old (95\% CI 36.51-52.47), which was significantly higher than the non-intensive ICU patients ( 45 vs 35 years old; $p=0.0062$ ). Kruskal Wallis analysis of the multiple variables of ICU patients compared to the non-intensive COVID-19 patients and the control group yielded many observed differences. Statistically significant differences were observed in the RBC indices as well as the platelet count $(p<0.0001)$. In fact, the median platelet count of ICU patients was significantly higher compared to non-intensive patients (249 vs $230 \times 10^{3} / \mu \mathrm{l} ; p<0.05$ ). However, both patient cohorts, non-intensive and ICU, had significantly lower platelet count compared to the control cohort (230 and 249 vs $\left.309.5 \times 10^{3} / \mu \mathrm{l} ; p<0.0001\right)$.

Table 2 - Clinical characteristics and laboratory results of the control and both patients' cohorts (non-intensive and intensive care unit [ICU]-admitted) Covid-19 patients.

\begin{tabular}{|c|c|c|c|c|c|}
\hline Characteristics & $\begin{array}{l}\text { Controls } \\
(\mathrm{n}=250)\end{array}$ & $\begin{array}{l}\text { Non-intensive } \\
\text { COVID-19 cases } \\
\quad(n=660)\end{array}$ & $\begin{array}{l}\text { ICU-admitted } \\
\text { COVID-19 patients } \\
(\mathrm{n}=41)\end{array}$ & $\begin{array}{c}P \text {-value } \\
\text { (Kruskal Wallis) }\end{array}$ & $\begin{array}{c}P \text {-value (between } \\
\text { non-intensive } \\
\text { and ICU) }\end{array}$ \\
\hline \multicolumn{6}{|l|}{ Gender, $n(\%)$} \\
\hline Male & $138(55.2)$ & $295(44.7)$ & $22(53.66)$ & \multirow{2}{*}{$0.013^{*}$} & \multirow{2}{*}{0.12} \\
\hline Female & $112(44.8)$ & $365(55.3)$ & $19(46.34)$ & & \\
\hline Age, years & $48(44.58-48.13)$ & $35(34.6-37.26)$ & $45(36.51-52.47)$ & $<0.0001^{*}$ & $0.0062^{*}$ \\
\hline Red blood cell count x $10^{6} / \mathrm{L}$ & $5.08(5.02-5.11)$ & $4.72(4.63-4.76)$ & $4.6(4.27-4.8)$ & $<0.0001^{*}$ & 0.37 \\
\hline Hemoglobin: g/dL & $14(13.65-14.06)$ & $13.6(13.01-14.25)$ & $13.2(12.09-13.35)$ & $0.0012^{*}$ & 0.067 \\
\hline Mean corpuscular fL & $90.35(88.74-90.8)$ & $86.4(84.9-86.34)$ & $86.3(83.35-88.14)$ & $<0.0001^{*}$ & 0.91 \\
\hline Mean corpuscular hemoglobin pg & $29.55(29.28-29.69)$ & $28.8(27.93-28.51)$ & $28.6(27.58-28.94)$ & $<0.0001^{*}$ & 0.63 \\
\hline $\begin{array}{l}\text { Mean corpuscular hemoglobin } \\
\text { concentration } \mathrm{g} / \mathrm{dL}\end{array}$ & $33(32.91-33.14)$ & $33(32.62-33.06)$ & $32.8(32.54-33.22)$ & 0.72 & 0.5 \\
\hline Platelet count $\times 10^{3} / \mu \mathrm{l}$ & $309.5(297.5-320.7)$ & $230(238.9-258.3)$ & $249(245.5-309.9)$ & $<0.0001^{*}$ & $0.046^{*}$ \\
\hline White blood cell count $\times 10^{3} / \mu \mathrm{l}$ & $7.2(6.92-7.37)$ & $5.37(5.9-6.58)$ & $6.96(6.96-10.29)$ & $<0.0001^{*}$ & $0.0008^{*}$ \\
\hline Neutrophil count $\times 10^{3} / \mu \mathrm{l}$ & $4.4(4.28-4.64)$ & $3.39(3.92-4.56)$ & $4.6(4.47-7.57)$ & $<0.0001^{*}$ & $0.0043^{*}$ \\
\hline Neutrophil percentage & $58(56.03-58.6)$ & $66.1(62.82-65.98)$ & $76.05(68.12-78.53)$ & $<0.0001^{*}$ & $0.0005^{*}$ \\
\hline Lymphocyte count $\times 10^{3} / \mu l$ & $2.1(1.95-2.1)$ & $1.2(1.25-1.46)$ & $1(0.92-1.63)$ & $<0.0001^{*}$ & 0.05 \\
\hline Lymphocyte percentage & $30.45(29.8-31.31)$ & $23.05(23.3-26.13)$ & $14.2(13.29-21.34)$ & $<0.0001^{*}$ & $0.0007^{*}$ \\
\hline Monocyte count $\times 10^{3} / \mu \mathrm{l}$ & $0.6(0.61-0.69)$ & $0.32(0.31-0.62)$ & $0.38(0.37-0.62)$ & $<0.0001^{*}$ & 0.17 \\
\hline Neutrophil-to-lymphocyte ratio & $2.18(2.3-2.6)$ & $2.85(3.89-4.9)$ & $5.5(5.87-11.66)$ & $<0.0001^{*}$ & $0.0014^{*}$ \\
\hline
\end{tabular}

Laboratory results are stated as median values with $95 \%$ confidence intervals between brackets, unless stated otherwise. * ${ }^{*}$ tatistical significance 
Significant differences were observed in the WBC indices between the control and 2 patients' cohorts. There were significant differences in the total WBC, as well as other indices such as, neutrophil and lymphocyte counts and percentages and NLR. Compared to non-intensive patients, ICU patients had significantly higher WBC count (6.96 vs $\left.5.37 \times 10^{3} / \mu \mathrm{l} ; p<0.0008\right)$, higher neutrophil count ( 4.6 vs $3.39 \times 10^{3} / \mu \mathrm{l} ; p<0.0043$ ) and lower lymphocyte count, although not significantly ( 1 vs $1.2 \times 10^{3} / \mu \mathrm{l} ; p=0.05$ ). Furthermore, ICU patients had significantly higher NLR, almost as twice as nonintensive patients (5.5 vs $2.85 ; p=0.0014$ ) as shown in Figure 2.

To examine whether the NLR correlated with any of the ICU patients' biological variables, multiple linear regression analysis was performed. Similar to non-intensive patients, lymphocytic count significantly correlated with NLR $(p<0.0001)$. Noticeably, a positive correlation was observed between NLR and the MCV among ICU patients $(p<0.05)$.

An overall analysis of the total patients' cohort has yielded an NLR value of 5.5 which has been tested. An

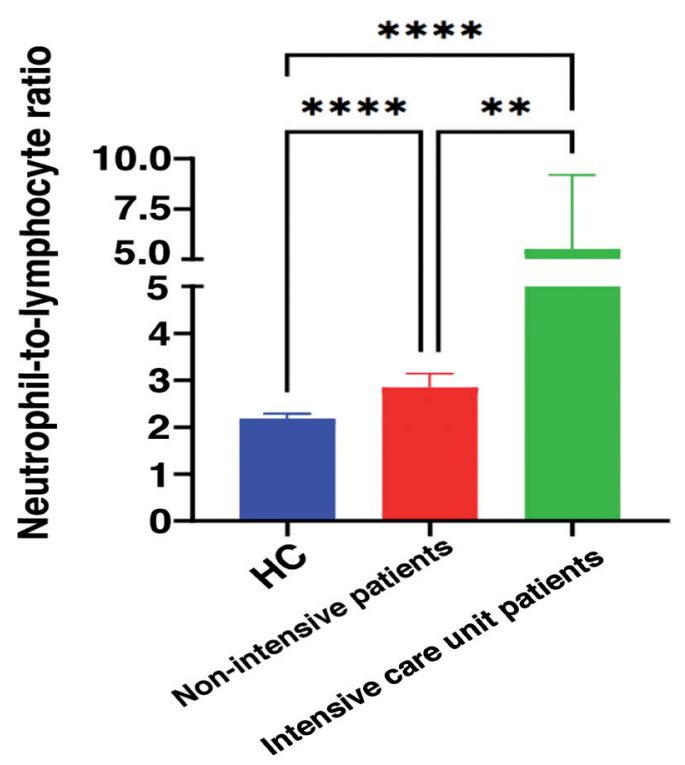

Figure 2 - Comparisons of neutrophil-to-lymphocyte ratios (NLR) between the different cohorts. The bar charts demonstrate the differences of the median NLR between the different cohorts. The chart shows that the control cohort (blue) which has the lowest NLR value compared to non-intensive patients (red), and the ICU patients (green). ICU patients have the highest NLR compared to the other cohorts. HC: healthy control; ICU: intensive care unit, ${ }^{* *}$ denotes a $p<0.01,{ }^{* * * *}$ denotes a $p<0.0001$.
NLR value of 5.5 seemed to be of very low sensitivity in detecting cases of COVID-19, as compared to the control group, with sensitivity of $23.65 \%$. However, this value yielded a high positive prognostic value at $91.43 \%$ and a very high specificity at $96.4 \%$. In other words, a person with an NLR of 5.5 or more is very likely to have a COVID-19 infection, whereas a person with an NLR of less than 5.5 can be excluded from having a COVID-19 infection. Moreover, the NLR value of 5.5 seemed to be a useful tool in differentiating between ICU and non-intensive COVID-19 patients. Using an NLR value of 5.5 to compare between ICU and non-intensive COVID-19 patients gave a very good sensitivity at $86.36 \%$ and specificity of $78.9 \%$. Moreover, the yielded negative predictive value was $92.9 \%$. In other words, confirmed COVID-19 patients with an NLR of 5.5 or more, should be observed carefully as they are more prone to become ICU patients, while those with an NLR less than 5.5 will most likely be non-intensive patients.

Discussion. Since the beginning of the COVID-19 pandemic, healthcare systems strived to develop diagnostic tools, as well as identifying prognostic markers as it is currently without a definitive treatment. Although it was initially thought to be a respiratory infection, increasing evidence have demonstrated its systematic manifestation indicating that it is more than just a respiratory condition. Provided its systematic effect, studies have started to identify prominent hematological changes that served as an independent indicator for the disease severity. Of these markers, NLR was found to be significantly higher in cases of COVID-19 infection, and an indicator of severe forms of the disease.

The aim of this pilot case-control study in Saudi Arabia was to compare the laboratory findings in patients with COVID-19 to healthy controls, as well as to assess the value of NLR in COVID-19 infection. In this study, patients had significantly lower hemoglobin levels and platelet count, which are in line with the results shown by Sun et al. ${ }^{13}$ Interestingly, the patients' cohort were significantly younger compared to the control group. The median age of patients in this study was 35 years old, which was similar to the national cohort previously described by Alsofayan et $\mathrm{al}^{14}$ as well as Al-Omari et al. ${ }^{15}$ However, the ICU cohort was significantly older than the non-intensive patients, confirming the works of Yi et $\mathrm{al}^{16}$ and Rosenthal et $\mathrm{al}^{17}$ that age was an independent risk factor for COVID-19 infection.

In our study, the NLR values of patients were significantly higher in patients compared to control 
group. Furthermore, it was even higher among ICU patients, as compared to both the control and nonintensive patients. Such findings are in line with the previous work of Yufei et $\mathrm{al}^{18}$ and Chan et $\mathrm{al}^{19}$ research groups. The value of NLR, being higher in ICU patients, serves as an independent indicator of disease severity, and could also serves as a prognostic tool for predicting poor prognosis upon admission. In this study, an NLR of 5.5 demonstrated a very high positive predictive value value of ICU admission, at $96.4 \%$. This value was higher than the NLR previously described by Tatum et $\mathrm{al}^{20}$ at 4.94 , in a cohort of 188 COVID-19 patients in Louisiana, United States. They estimated that COVID-19 patients with an NLR of 4.94 at significantly higher odds of longer ICU admission and in-hospital intubation, as well as a strong prognostic factor for increased mortality. ${ }^{20}$ In fact, similar findings from a Chinese study led by Liu et $\mathrm{al}^{21}$ concluded that higher NLR values are associated with higher risk for in-hospital deaths. Importantly, the value of NLR as a prognostic factor of severe COVID-19 infections is independent from any other risk factors such as age or gender. In our study, the median age of the patients' cohort was 35 years old, as compared to other studies in which the median ages of patients were 53, 58.7 and 62 years. Regardless of the participants' age, in ours or other studies, higher NLR values $(\geq 4)$ was a risk factor for severe COVID-19 infections indicated by ICU admission, mechanical ventilation and mortality rates..$^{20,22,23}$

Study limitations. Although every effort was carried out to execute this study in a proper manner, this study is not without limitation. In order to validate the predictive value of NLR, ideally it should be tested longitudinally in a prospective study. In other words, the NLR should be determined upon admission and patients are to be followed up to assess how accurate the NLR value in determining the disease outcome. The control group $(n=250)$ in this study were patients who were admitted for elective surgical procedures. Although they tested negative for COVID-19 infection and were fit for the scheduled procedure, other infections and comorbidities were not ruled out. Ideally, the control group should be consisted of healthy volunteers, in a number that is close to tested cohort, and should not have any infections nor comorbidities that may influence the NLR.

In conclusion, NLR is an important tool in determining the status of the COVID-19 infection. Although its sole use in COVID-19 diagnosis may not be very accurate, its use in conjunction with a proper history-taking and physical examination should be very useful in ruling out the infection. Furthermore, the findings of this study further confirm that NLR is an independent prognostic factor of a severe infection, and those patients with high NLR should be closely monitored and managed.

Acknowledgment. The authors express their thanks and gratitude to Ms. Fong H. Yuen for her support and assistance in the data collection process. The authors would also like to thank Scribendi (www.scribendi.com) for English language editing.

\section{References}

1. Chen N, Zhou M, Dong X, Qu J, Gong F, Han Y, et al. Epidemiological and clinical characteristics of 99 cases of 2019 novel coronavirus pneumonia in Wuhan, China: a descriptive study. Lancet 2020; 395: 507-513.

2. Kerboua KE. NLR: A cost-effective nomogram to guide therapeutic interventions in COVID-19. Immunol Invest 2020; 50: 92-100.

3. Guan W, Ni Z, Hu Y, Liang W, Ou C, He J, et al. Clinical characteristics of coronavirus disease 2019 in China. N Engl J Med 2020; 382: 1708-1720.

4. Xu Z, Shi L, Wang Y, Zhang J, Huang L, Zhang C, et al. Pathological findings of COVID-19 associated with acute respiratory distress syndrome. Lancet Respir Med 2020; 8: 420-422.

5. Wang P, Anderson N, Pan Y, Poon L, Charlton C, Zelyas N, et al. The SARS-CoV-2 outbreak: diagnosis, infection prevention, and public perception. Clin Chem 2020; 66: 644-651.

6. Wu Z, McGoogan JM. Characteristics of and important lessons from the Coronavirus disease 2019 (COVID-19) outbreak in China. JAMA 2020; 323: 1239.

7. Huang Z, Fu Z, Huang W, Huang K. Prognostic value of neutrophil-to-lymphocyte ratio in sepsis: a meta-analysis. $\mathrm{Am} \mathrm{J}$ Emerg Med 2020; 38: 641-647.

8. Templeton AJ, McNamara MG, Šeruga B, Vera-Badillo FE, Aneja P, Ocańa A, et al. Prognostic role of neutrophil-tolymphocyte ratio in solid tumors: a systematic review and metaanalysis. J Natl Cancer Inst 2014; 106: dju124.

9. Wang Y, Ju M, Chen C, Yang D, Hou D, Tang X, et al. Neutrophil-to-lymphocyte ratio as a prognostic marker in acute respiratory distress syndrome patients: a retrospective study. $J$ Thorac Dis 2018; 10: 273-282.

10. Liu J, Liu Y, Xiang P, Pu L, Xiong H, Li C, et al. Neutrophilto-lymphocyte ratio predicts critical illness patients with 2019 coronavirus disease in the early stage. J Transl Med 2020; 18 : 206.

11. Ministry of Health. Weqaya Saudi Center for Disease Prevention and Control. Coronavirus Disease 19 (COVID-19) Guidelines V1.1. [Updated 2020 February. 2020 September 21]. Available from URL: https://www.moh.gov.sa/CCC/healthp/regulations/ Documents/Coronavirus Disease 2019 Guidelines v1.1..pdf

12. Dolgin M, The Criteria Committee of the New York Heart Association. Nomenclature and criteria for diagnosis of diseases of the heart and great vessels. Nomenclature and Criteria for Diagnosis of Diseases of the Heart and Great Vessels. Boston NY: Little Brown; 1994.

13. Sun S, Cai X, Wang H, He G, Lin Y, Lu B, et al. Abnormalities of peripheral blood system in patients with COVID-19 in Wenzhou, China. Clin Chim Acta 2020; 507: 174-180. 
14. Alsofayan YM, Althunayyan SM, Khan AA, Hakawi AM, Assiri AM. Clinical characteristics of COVID-19 in Saudi Arabia: A national retrospective study. J Infect Public Health 2020; 13: 920-925.

15. Al-Omari A, Alhuqbani WN, Zaidi ARZ, Al-Subaie MF, AlHindi AM, Abogosh AK, et al. Clinical characteristics of non-intensive care unit COVID-19 patients in Saudi Arabia: A descriptive cross-sectional study. J Infect Public Health 2020; 13: 1639-644.

16. Yi P, Yang X, Ding C, Chen Y, Xu K, Ni Q, et al. Risk factors and clinical features of deterioration in COVID-19 patients in Zhejiang, China: a single-centre, retrospective study. $B M C$ Infect Dis 2020; 20: 943.

17. Rosenthal N, Cao Z, Gundrum J, Sianis J, Safo S. Risk Factors Associated With In-Hospital Mortality in a US National Sample of Patients With COVID-19. JAMA Netw Open 2020; 3: e2029058.

18. Yufei Y, Mingli L, Xuejiao L, Xuemei D, Yiming J, Qin Q, et al. Utility of the neutrophil-to-lymphocyte ratio and C-reactive protein level for Coronavirus disease 2019 (COVID-19). Scand J Clin Lab Invest 2020; 80: 536-540.
19. Chan AS, Rout A. Use of neutrophil-to-lymphocyte and platelet-to-lymphocyte ratios in COVID-19. J Clin Med Res 2020; 12: 448-453.

20. Tatum D, Taghavi S, Houghton A, Stover J, Toraih E, Duchesne J. Neutrophil-to-lymphocyte ratio and outcomes in Louisiana COVID-19 Patients. Shock 2020; 54: 652-658.

21. Liu Y, Du X, Chen J, Jin Y, Peng L, Wang HHX, et al. Neutrophil-to-lymphocyte ratio as an independent risk factor for mortality in hospitalized patients with COVID-19. J Infect 2020; 81: e6-e12.

22. Zhang B, Zhou X, Zhu C, Song Y, Feng F, Qiu Y, et al. Immune phenotyping based on the neutrophil-to-lymphocyte ratio and IgG level predicts disease severity and outcome for patients with COVID-19. Front Mol Biosci 2020; 7: 157.

23. Kong M, Zhang H, Cao X, Mao X, Lu Z. Higher level of Neutrophil-to-Lymphocyte is associated with severe COVID-19. Epidemiol Infect 2020; 148: e139. 